\title{
2-Octyl Cyanoacrylate Skin Adhesive for Topical Skin Incision Closure in Female Pelvic Surgery
}

\author{
Rebecca L. James*, Marilyn Alejandro-Rodriquez, Elba Adriana Perez, Jeffrey Mangel \\ MetroHealth Medical Center, Cleveland, $\mathrm{OH}$, USA \\ Email: rebecca.james@uhhospitals.org
}

Received 7 April 2015; accepted 25 May 2015; published 27 May 2015

Copyright (C) 2015 by authors and Scientific Research Publishing Inc.

This work is licensed under the Creative Commons Attribution International License (CC BY). http://creativecommons.org/licenses/by/4.0/

(c) (i) Open Access

\begin{abstract}
Background: 2-octyl cyanoacrylate (20CA) high viscosity tissue adhesive (Medline Industries, Inc., Mundelein, IL) is a liquid topical skin adhesive. 20CA offers the same design features and clinical utility in terms of flexibility, strength, and low complication rate as the commercially available 20CA tissue adhesives. Additionally, 20CA features high viscosity allowing for better control during the application process, polymerization without the use of an external activator, and a distinctive violet color for ease of application visualization. Objective: The aim of this prospective case series is to descriptively report clinical data with the application of 20CA as a topical incision closure system in female pelvic surgery. The primary outcomes included: incisional pain, incisional dehiscence, and post-operative bleeding. The secondary outcome included drying times of the adhesive with regard to incision length. Methods: A prospective open-label observational case series study was conducted to evaluate the use of 20CA in surgical wound closure of the topical skin in adult patients undergoing gynecologic surgical procedures. A total of 50 adult women undergoing gynecologic surgery were enrolled. 20CA was applied only by surgeons who had undergone product training to the incisions in a standardized, protocol-defined fashion. Drying times for the adhesive and photography were recorded intraoperatively. Post treatment follow-up was conducted with queries of pain level, incisional dehiscence, and incisional bleeding immediately post-operatively, 48 hours, 5 - 10 days and 14 days post-treatment. Adverse events were documented. Results: 20CA was applied to a total of 154 incisions from the 50 patients enrolled to the study. The procedures included: 16 laparoscopic total hysterectomies, 4 diagnostic laparoscopies, 2 laparoscopic myomectomies, 2 laparoscopic bilateral or unilateral salpingo-oophorectomies, 5 total roboticassisted laparoscopic surgeries ( 2 total hysterectomies, 1 supracervical hysterectomy, 1 sacrocolpopexy, and one excision of endometriosis), 7 sacral neuromodulation procedures, and 18 midurethral slings. The overall rate of incisional dehiscence was $3 \%(4 / 154)$. The rate of reported incisional bleeding was $3 \%(4 / 154)$. There was 1 incisional infection. The pain reports based on a
\end{abstract}

\footnotetext{
*Corresponding author.
} 
10-point scale had a mean of 4.96 immediately post-operatively, which decreased to a mean score $<1(0.2)$ by post-op day (POD) 14. The mean drying times for the various lengths of incisions included the following: 1.28 minutes for incisions $\leq 5 \mathrm{~mm}, 1.53$ minutes for $6-8 \mathrm{~mm}, 1.66$ minutes for $10 \mathrm{~mm}-20 \mathrm{~mm}$, and 1.57 minutes for the $40-50 \mathrm{~mm}$ incisions. In $23 \%$ (36/154) of incisions 20CA was the sole method of skin closure. Conclusion: This study demonstrates that 20CA is safe to use in gynecologic surgical incisions with low rates of post-operative incisional bleeding and incisional dehiscence. Post-operative reports of pain maintained expected levels for recovery. 20CA is a practical alternative or augmentation to traditional suture closure of skin incisions at the time of gynecologic surgery.

\section{Keywords}

Female Pelvic Surgery, Gynecologic Surgery, Octyl Cyanoacrylate, Surgical Incision, Skin Closure

\section{Background}

2-octyl cyanoacrylate (2OCA) high viscosity tissue adhesive (Medline Industries, Inc., Mundelein, IL) is a liquid topical skin adhesive. 2OCA offers the same design features and clinical utility in terms of flexibility, strength, and low complication rate as the commercially available 2OCA tissue adhesives. Additionally, 2OCA features high viscosity allowing for better control during the application process, polymerization without the use of an external activator, and a distinctive violet color for ease of application visualization. The adhesive film generally sloughs off within 5 to 10 days, so there is no need for the patient or provider to remove the adhesive [1]. Extensive mechanical and in vitro performance characterizations have been conducted on 2OCA, as well as in vivo incision studies in animal models [2].

\section{Objective}

The aim of this prospective case series is to descriptively report clinical data with the application of 2OCA as a topical incision closure system in female pelvic surgery. The primary outcomes included: incisional pain, dehiscence, and post-operative bleeding. The secondary outcome included drying times of the adhesive with regard to incision length.

\section{Methods}

After institutional review board approval, a prospective open-label observational case series study was conducted in the Divisions of Urogynecology and Minimally Invasive Surgery to evaluate the application of 2OCA in surgical topical incision closure of the skin in women undergoing female pelvic surgery. Primary outcomes included: incisional pain, dehiscence, and post-operative bleeding. Secondary outcomes included drying times of the adhesive with regard to incision length.

Patients were approached for recruitment either in the outpatient setting when preparing for scheduled surgery or pre-operatively on the date of surgery. Inclusion criteria included women greater than or equal to age 18 who had scheduled female pelvic surgery procedures and had who never previously had 2OCA applied. Intraoperatively, 2OCA was applied to the skin incision(s) in the standardized method taught during surgeon training by Medline prior the study initiation. The technique included approximation of the skin edges with forceps and application of the first layer of 2OCA using the applicator. After at least 30 seconds of drying, a second layer of 2OCA was applied. Drying times were recorded from the start of the second layer of adhesive until gentle touching via the forceps concluded a completely dried seal. Additionally, de-identified photography was taken of each patient's skin incisions in the operating room (See Figure 1 and Figure 2).

A series of 4 visiting time points included: bedside POD 0 during recovery and follow-up phone calls (FUV) 48 hours after surgery, 5 - 10 days after surgery, and POD 14. These visiting time points included asking the patient to rate her pain on a $1-10$ scale (with a visual analog scale provided during the recovery visit POD 0 ) as well as queries of incision dehiscence (defined as skin separation) and active bleeding. These POD 0 visits were 


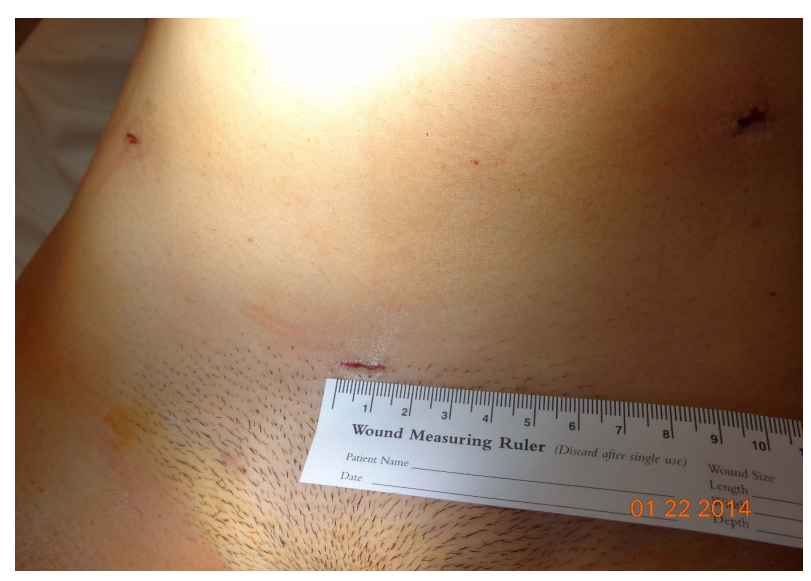

Figure 1. 2OCA applied to laparoscopic incision.

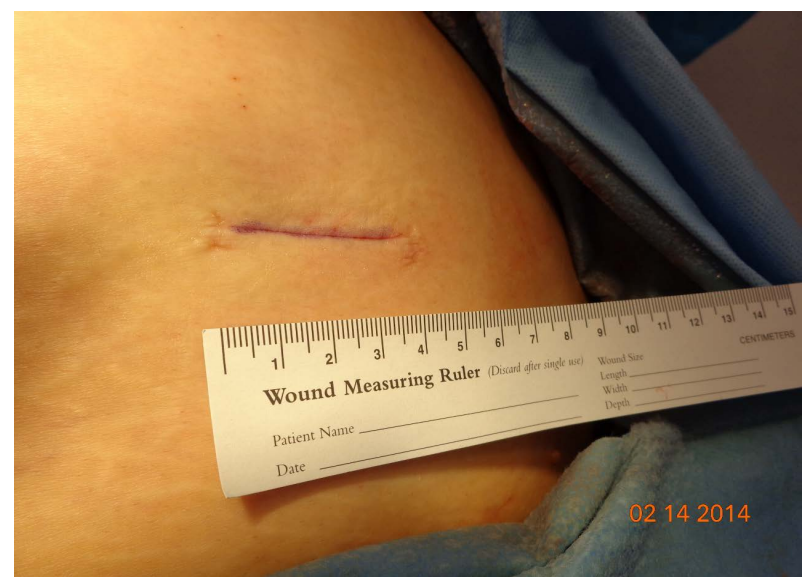

Figure 2. 2OCA applied to a sacro neuromodulation incision.

completed primarily by the first author (often the fellow co-surgeon during the surgical cases), a senior fellow co-surgeon, and one bedside visit by our research nurse (of which the findings were then also confirmed by the first author). The remaining FUV by phone were conducted by the first author and research nurse. During the FUV, the patients were asked to again rate the level of pain on 1 - 10 scale. They were also asked if any incision separation or bleeding had occurred. For those whose incisions were located on their back, patients reported using home mirrors to assess for any separation or bleeding. Data including demographic information, type of surgery, patient comorbidities, locations of incisions drawn and numbered on a female diagram, outcomes, and any adverse events with any respective treatments. These data were recorded first on standardized data collection sheets provided by Medline and then uploaded and managed within a RedCap ${ }^{\mathrm{TM}}$ database hosted by Case Western Reserve University ${ }^{1}$.

\section{Results}

Of 56 women approached, 50 patients agreed to enroll in the study. 2OCA was applied to a total of 154 incisions from these 50 patients. Mean age was 46.3 with age ranging from 29 - 79. There were 19 African American patients, 24 Caucasian patients, and 7 Hispanic patients. The procedures included: 16 laparoscopic total hysterectomies, 4 diagnostic laparoscopies, 2 laparoscopic myomectomies, 2 laparoscopic bilateral or unilateral salpingo-oophorectomies, 5 total robotic-assisted laparoscopic surgeries (2 total hysterectomies, 1 supracervical hysterectomy, 1 sacrocolpopexy, and 1 excision of endometriosis), 7 sacral neuromodulation procedures, and 18

${ }^{1}$ REDCap (Research Electronic Data Capture) is a secure, web-based application designed to support data capture for research studies, providing 1) an intuitive interface for validated data entry; 2) audit trails for tracking data manipulation and export procedures; 3) automated export procedures for seamless data downloads to common statistical packages; and 4) procedures for importing data from external sources. 
midurethral slings (see Table 1). The overall rate of incisional dehiscence was 3\% (4/154) (Table 2). One patient with an incisional dehiscence experienced separation of a $10 \mathrm{~mm}$ suprapubic incision that was under a heavy pannus on POD 0 while inpatient. A single interrupted suture with local injection of anesthesia performed at the bedside easily corrected the separation, and our surgical team has considered that the heaviness of the pannus and location of the incision more likely contributed to this separation rather than the 2OCA. The other three incisions with dehiscence occurred on POD 2 and were easily resolved by application of cover dressings for protection per nurse visit evaluation. The rate of reported incisional bleeding was 3\% (4/154) (Table 2). There was 1 incisional infection based on erythema seen in the office, and this patient received empiric antibiotics. The pain reports based on a 10-point scale had a mean of 4.96 immediately post-operatively, which decreased to a mean score $<1(0.2)$ by post-op day (POD) 14 . The mean drying times for the various lengths of incisions included the following: 1.28 minutes for incisions $\leq 5 \mathrm{~mm}(\mathrm{n}=57), 1.53$ minutes for $6-8 \mathrm{~mm}(\mathrm{n}=19)$, 1.66 minutes for $10 \mathrm{~mm}-20 \mathrm{~mm}(\mathrm{n}=71)$, and 1.57 minutes for the $40-50 \mathrm{~mm}$ incisions $(\mathrm{n}=7)$ (see Table 3 ). Other adverse events included vaginal odor, which was reported in the study, but was not attributed to be associated with 2OCA. In 23\% (36/154) of incisions 2OCA was the sole method of skin closure. Given the variety of number of incisions per patient and depending on the surgery type, between $1-2$ applicators yielded sufficient

\begin{tabular}{lc}
\hline Table 1. List of gynecologic surgeries by type. \\
\hline \multicolumn{1}{c}{ Type of Gynecologic Surgery } & Number of Procedures \\
\hline Total laparoscopic hysterectomy & 16 \\
Diagnostic laparoscopy & 4 \\
Laparoscopic myomectomy & 2 \\
Laparoscopic salpingo-oophorectomy & 2 \\
Robotic assisted surgery n = 5 & \\
Total hysterectomy & 2 \\
Supracervical hysterectomy & 1 \\
Sacrocolpopexy & 1 \\
$\quad$ Excision of endometriosis & 1 \\
Midurethral sling & 18 \\
Sacroneuromodulation (Stages I or II) & 7 \\
\hline
\end{tabular}

Total incisions $\mathrm{n}=154$.

Table 2. Adverse event rates.

\begin{tabular}{cccc}
\hline & Infection & Dehiscence & Bleeding \\
\hline Surgiseal $n=154$ & $1 / 154(<1 \%)$ & $4 / 154(3 \%)$ & $4 / 154(3 \%)$ \\
Dermabond $n=228$ & $8 / 228(3.6 \%)$ & $59 / 228(24.9 \%)$ & $\begin{array}{c}\text { Cannot be determined } \\
\text { from literature }\end{array}$ \\
\hline
\end{tabular}

Table 3. Incision drying times.

\begin{tabular}{ccc}
\hline Incision length & Drying time in minutes (2 layers) & $\mathrm{n}=154$ total \\
\hline$\leq 5 \mathrm{~mm}$ & 1.28 & 57 \\
$6-8 \mathrm{~mm}$ & 1.53 & 19 \\
$10-12 \mathrm{~mm}$ & 1.66 & 71 \\
$40-50 \mathrm{~mm}$ & 1.57 & 7 \\
\hline
\end{tabular}


product to apply two layers of 2OCA according to protocol. Of note, during phone visits two patients volunteered commentary about desiring to use 2OCA again if ever undergoing further surgery, although the question was never formally posed since it was not within the protocol. Additionally, 2 patients who required additional surgery due to the staged procedures of sacral neuromodulation asked to have 2OCA applied to their incisions again, although we could not offer it a second time within the study given the protocol.

\section{Conclusion}

This study demonstrates that 2OCA is safe to use in gynecologic surgery from incision closure, post-operative bleeding, and pain standpoints. Post-operative reports of pain maintained expected levels for recovery. Rapid drying times offer suitable alternatives to use of suture when closing incisions at the completion of cases. Future trials comparing traditional skin suture with skin adhesive closures within minimally invasive female pelvic surgery are necessary to continue investigations of cosmesis, efficacy, and intraoperative efficiency. 2OCA is a practical alternative or augmentation to traditional suture closure of skin incisions at the time of gynecologic surgery.

\section{Discussion}

A skin incision closure technique should ideally be safe, easy to use, rapid, aesthetic, cost-effective, and not require device removal [3]. While numerous publications exist in the realm of skin adhesives for closure of surgical incisions, few exist related to gynecologic surgery and minimally invasive female pelvic surgery.

We intend to discuss the adverse event rate comparisons between a traditional 2OCA product which has been reported in literature and those of the newer product in this study. It is now known that 2OCA require an initiator for polymerization to form the adhesive bond to hold the wound edges together. Such initiators can be located either within the liquid itself or at the tip of the dispensing end of the applicator. In either case, the steps taken to activate the applicator also activate the action of the initiator to the cyanoacrylate monomer. Two products are described going forth: one in which the applicator tip contains the initiator, 2 OctylCyanoAcrylate with Tip Initiator (2OCATI), and the other described in this study where the initiator is present in the monomer itself, 2 OctylCyanoAcrylate with Internal Initiator (2OCAII).

With regard to larger gynecologic incisions, Murrmann et al. retrospectively compared 2OCATI with conventional suture or staple closure of women undergoing total abdominal hysterectomy. Sutures compared to 2OCATI showed no difference in length of inpatient stay, mean total cost, or antibiotics treatment [4]. Laparoscopic port site incisions were closed faster by surgical adhesive than sutures in a metaanalysis of seven trials [5]. Among general surgery laparoscopic procedures, a randomized controlled trial of n-butyl cyanoacrylate tissue adhesive versus sutures showed significantly shorter incision closure times and no differences in wound complications or cosmesis at either 4 to 6 weeks or at 3 months [6]. A study from Poland reported no difference in method of healing and local tissue reaction between skin adhesive and sutures in laparoscopic incisions, however the cosmetic result was significantly improved with skin adhesive [7]. Mean skin closure time was reported to be 14.5 seconds in incisions measuring 2 to $6 \mathrm{~cm}$ with use of cyanoacrylate during a study of laparoscopic tubal ligations, however only one layer of adhesive was applied [8]. While the mean drying times in our study on incisions ranged from 1.28 minutes for incisions $5 \mathrm{~mm}$ or less to 1.66 minutes for 10 - $20 \mathrm{~mm}$, our protocol involved the application of two adhesive layers with complete drying of the first layer. A randomized, doublearmed trial of 2OCATI applied to laparoscopic incisions showed mean skin closure time of 3.7 minutes in the adhesive group requiring 3.4 vials per patient, a significant decrease compared to 14 minutes in the suture arm. Additionally, a mean decrease of 9 minutes of operative time per case resulted in significant cost savings when calculated by operating room time fees [9]. In terms of adverse events, 2OCATI had wound dehiscences in 5/228 laparoscopic incisions (2.19\%), 1 surgical site infection (0.44\%), and 3/228 seromas (1.32\%) [9]. The wound separation rate for 2OCATI ranged from $0.78 \%-1.18 \%$, in a study of 228 wounds, which is similar to the package insert range of $0 \%-1.8 \%$ when combined with suture or $2.1 \%-2.5 \%$ without sutures [9]-[11]. The infection rate was reported to be $3.6 \%$ the study of 228 wounds [11]. The adverse event rates of 2OCAII in this study were comparable in our population of 154 incisions, as detailed in Table 3 [10] [11]. Our preliminary results in the population of minimally invasive female pelvic surgery patients appear to follow the trend in literature of surgical adhesives showing encouraging safety profiles and efficient closure times. The safety profiles of 2OCATI and 2OCAII seem to be similar in regard to adverse events comparisons [10] [11]. The types of inci- 
sions in the 2OCATI study included $47.8 \%$ skin lesion excisions, $27.4 \%$ minimally invasive surgery punctures, and $24.8 \%$ general surgery incisions [10]. Our study was comprised of a variety of minimally invasive incision lengths (Table 2), with $46 \%$ in the $10-12 \mathrm{~mm}$ length. Specifically, the application of 2OCAII applied to the smaller incisions of midurethral slings or the upper lateral buttock 40 - $50 \mathrm{~mm}$ incisions of sacral neuromodulation has not previously been studied in literature reports, so these incision types offer a unique set of results. Future comparison trials between traditional skin suture and 2OCA closures within minimally invasive female pelvic surgery are necessary to continue investigations of cosmesis, efficacy, and intraoperative efficiency.

\section{Acknowledgements}

Special acknowledgements to Bogdan Orasanu, MD, Robert Pollard, MD, and Sarah Kane, MD for contributing surgical patients.

\section{References}

[1] Singer, A.J., Quinn, J.V. and Hollander, J.E. (2008) The Cyanoacrylate Topical Skin Adhesives. The American Journal of Emergency Medicine, 26, 490-496. http://dx.doi.org/10.1016/j.ajem.2007.05.015

[2] (2014) Monograph on Surgiseal ${ }^{\circledR}$ Topical Skin SS035T: Surgiseal (Teardrop) and STC50S: Surgiseal Twist ${ }^{\mathrm{TM}}$.

[3] Singer, A.J., Hollander, J.E. and Quinn, J.V. (1997) Evaluation and Management of Traumatic Lacerations. The New England Journal of Medicine, 337, 1142-1148. http://dx.doi.org/10.1056/NEJM199710163371607

[4] Murrmann, S.G., Markowitz, J.S., Gutterman, E.M. and Magee, G. (2010) Postoperative Outcomes Associated with Topical Skin Adhesives among Women Having Hysterectomies. Surgical Infections, 11, 441-447. http://dx.doi.org/10.1089/sur.2009.032

[5] Sajid, M.S., Siddiqui, M.R., Khan, M.A. and Baig, M.K. (2009) Meta-Analysis of Skin Adhesives versus Sutures in Closure of Laparoscopic Port-Site Wounds. Surgical Endoscopy, 23, 1191-1197. http://dx.doi.org/10.1007/s00464-009-0373-y

[6] Dowson, C.C., Gilliam, A.D., Speake, W.J., Lobo, D.N. and Beckingham, I.J. (2006) A Prospective, Randomized Controlled Trial Comparing n-Butyl Cyanoacrylate Tissue Adhesive (LiquiBand) with Sutures for Skin Closure after Laparoscopic General Surgical Procedures. Surgical Laparoscopy, Endoscopy \& Percutaneous Techniques, 16, 146150. http://dx.doi.org/10.1097/00129689-200606000-00005

[7] Szyllo, K., Wlodarczyk, B., Krekora, M., Lewy, J., Oszukowski, P. and Wilczynski, J. (2002) Application of Skin Glue in Wound Repair after Laparoscopy. Ginekologia Polska, 73, 567-570.

[8] Gorozpe Calvillo, J.I., Gonzalez-Villamil, J., Santoyo-Haro, S. and Castaneda-Vivar, J.J. (1997) Closure of the Skin with Cyanoacrylate in Surgical Wounds after Tubal Sterilization. Ginecologia y Obstetricia de Mexico, 65, 64-67.

[9] Sebesta, M.J. and Bishoff, J.T. (2004) Octylcyanoacrylate Skin Closure in Laparoscopy. JSLS: Journal of the Society of Laparoendoscopic Surgeons/Society of Laparoendoscopic Surgeons, 8, 9-14.

[10] (2009) Instruction for Use, High Viscosity Dermabond, PM 772334B, (C) Ethicon Inc.

[11] Hall, L.T. and Bailes, J.E. (2005) Using Dermabond for Wound Closure in Lumbar and Cervical Neurosurgical Procedures. Neurosurgery, 56, 147-150. http://dx.doi.org/10.1227/01.NEU.0000144170.39436.52 